\title{
The study of methods for combined processing of deep holes of hydraulic cylinders
}

\author{
Meruert A. Berikbaeva ${ }^{1}$, Beibyt T. Khairullin ${ }^{1}$, Railya M. Mukhamadeyeva ${ }^{2}$ \\ ${ }^{1}$ M. Kozybayev North Kazakhstan State University \\ 150000, 114 Zhumabaev Str., Petropavlovsk \\ ${ }^{2}$ Abay Myrzakhmetov Kokshetau University \\ 020000, 189a M. Auezov Str., Kokshetau \\ Republic of Kazakhstan
}

Received: July 22, 2020. Revised: September 23, 2020. Accepted: October 12, 2020. Published: October 19, 2020.

\begin{abstract}
Obtaining of the quality of the inner surface of the hydraulic cylinders and its optimization is an urgent problem in the enterprise. The article presents the results of a study to improve the finishing process of deep holes in thin-walled sleeves. The authors used the combination of the cutting process and surface plastic deformation for improving metal processing. The selected main parameters for ensuring the quality of the surface are roughness index and the value of internal stresses in the surface layer of hydraulic cylinders. To determine the hardening depth of the inner surface of the deep hole of the liner, the oblique thin section method was used. A mathematical model of the combined processing was obtained. The research results make it possible to recommend combined hole processing that combines cutting and plastic deformation. The results obtained in this work will improve the processing of deep holes of hydraulic cylinders, and the developed mathematical model has practical and theoretical scientific value.
\end{abstract}

Keywords - combined processing, hardening of the surface layer, processing of deep holes, roughness of the inner surface of hydraulic cylinders.

\section{INTRODUCTION}

A $\mathrm{N}$ urgent problem in the enterprise is to obtain the quality of the inner surface of the hydraulic cylinders and its optimization in order to ensure the required operational properties of the surfaces of parts and machines and devices in general. The connection between the surface layer quality characteristics and the operational properties of the parts indicates that the optimum (from the point of view of increasing the operational properties of the parts) surface should be sufficiently solid, should have compressive residual stresses, a finely dispersed structure, and a smoothed shape of microroughnesses with a large supporting surface area [1].

Hydraulic cylinders are common mechanisms. They are used in almost all types of special equipment, for example, hydraulic cylinders are used in excavators, truck cranes, bulldozers, aerial platforms, cranes, manipulators, concrete pumps, loaders, compressors, dump trucks. In principle, hydraulic cylinders are similar to pneumatic cylinders, however, the driving force in the hydraulic cylinder is a liquid, such as water or oil. Wear resistance is the main requirement for the inner surface of the hydraulic cylinder. The wear resistance of the inner bore of the sleeve will depend on (i) the waviness and roughness (more precisely, on the height of the scallops of irregularities), (ii) the actual contact surface area, (iii) the pitch and shape of individual irregularities, and (iv) the microhardness of the surface layer. Thin and numerous bumps provide greater wear resistance than large bumps. According to experimental data of Prof. P.E. Dyachenko [2], with liquid friction and a small height of irregularities, the direction of the processing pattern does not matter. When finishing the openings of hydraulic cylinders, the phenomenon of hardening that accompanies cold plastic deformation (CPD) is of primary interest (both theoretically and in practice). In our case, the optimum is a surface having residual stresses in the range of 200-350 MPa and an increase in microhardness of at least 15\% [3].

The researches of Prof. P.I. Yashcheritsin [4] showed the effect of surface roughness of the workpiece on the performance properties of the part. Maximum wear resistance is inherent in samples with high-quality pre-treatment. Moreover, the so-called technological heredity takes place, i.e., change in the operational properties of parts under the influence of the technology of their manufacture. Technological heredity is manifested not only in the influence of the method and processing mode used in recent finishing operations. It can also be manifested in a change in the properties or loss of accuracy of the shape of the finished part during its operation as a result of the influence of certain surface quality elements created in 
the surface layer of the part when roughing.

For the appropriate use of the phenomenon of technological heredity, it is necessary to establish direct relationships between the operational characteristics of the parts and the processing conditions of the part in the main operations of its manufacture. To do this, it is most reasonable to establish a mathematical relationship: "operational characteristic-surface quality function", "surface quality-processing mode function". A joint solution will allow us to establish a direct relationship: "operational characteristic-processing mode". As indicated earlier, surface plastic deformation (SPD) treatment is more acceptable to provide the required performance characteristics. Five-hole machining methods have been selected: (i) vibration processing, (ii) impact rolling, (iii) durning (drawing), (iv) smoothing, and (v) rolling.

Rolling is considered to be a highly efficient way of machining holes that have a low roughness value. This method assumes processing using rolls that have a roller or ball structure. Rolling is necessary to compact the metal surface by applying pressure and rolling motion. With this method, the surface of the layer is hardened, and the hardness and wear resistance are increased. The second common method is vibration processing. This treatment refers to mechanical or chemical-mechanical processing of parts and workpieces. The process takes place by smoothing microroughnesses and removing material particles from the treated surface with particles of an abrasive medium that vibrates under the action of vibration of the chamber in which the processed parts and the working medium are located. This treatment is carried out in a dry environment or in a solution, which can contain various chemical additives.

Smoothing is another method. During the smoothing process, the tool is pressed with a certain force against the surface to be treated. Smoothing can be used to process external and internal surfaces of revolution, end surfaces on turning, carousel, drilling, boring and other machines. The next method is impact rolling. This method is characterized by the impact interaction of the rollers with the processed surface when it passes the protruding support elements. The last common method is drawing. This method assumes machining without removing the chips. In the drawing process, the rigid mandrel working tool moves into the workpiece hole with an interference fit.

Given the specific processing conditions, the production program and the requirements for the parts, we can say that only two operations are acceptable: rolling and smoothing. Under these conditions, in the manufacture of parts made of plastic steel 35, the best (from the point of view of wear resistance of hydraulic equipment parts) processing methods turned out to be those creating the greatest hardening of the surface layer metal. In this case, the surface roughness is less important than hardening of the metal of the surface layer. However, it should be noted that in cases where the degree of hardening of the metal of the surface layer for different types of processing is close, and the surface roughness is significantly different, the types of processing that create less rough surfaces give less wear.

From the above considerations, a lot of wear on the run-in parts seems unexpected. Microscopic examination made it possible to detect cracks on their surface that arise with reriveting of the metal of the surface layer. High wear resistance of parts processed by smoothing is explained by the presence of negative residual stresses [5]. For parts of hydraulic equipment with a long length, processing accuracy along the entire hole is of no small importance. All other things being equal, the change in size when ironing and rolling depends on the initial roughness and is approximately determined by the following formula:

$$
\Delta d \approx 2 / 3(R Z \text { init. }-R Z)
$$

where $\mathrm{R}_{\text {Zinit. }}$ and $\mathrm{R}_{\mathrm{Z}}$ are, respectively, the heights of the bumps before and after processing.

This formula provides quite acceptable accuracy provided that the initial roughness does not exceed the height of the bumps (respectively, not higher than $R_{a}=0.63$ ). Studies, the results of which are given in the book of V.M. Torbilo [6], confirm that smoothing and rolling during elastic contact of the workpieces and the tool practically do not change the accuracy of the initial shape of the workpiece, as well as the accuracy of the dimensions [7-11]. This means that such a characteristic as waviness remains uncorrected and significantly reduces the wear resistance of the sleeves. Fixing the tool must be rigid to fix the shape.

The high rigidity of the tool-blank system in a radial section when working with SPD tools provides high sensitivity of this system to the most insignificant fluctuations of tightness - the main processing parameter. This, in turn, leads to the formation of areas with different surface qualities - the so-called "spotting" [12; 13]. Material heterogeneity is unavoidable in an industrial environment. To exclude tightness fluctuations when encountering hard or soft inclusions, pre-treatment should exclude a large difference in the height of irregular scallops and correct macrogeometry defects. Increased requirements for preobtained hole are the main drawback of SPD methods. This drawback can be eliminated by combining the operations of preliminary processing by cutting with subsequent calibratingfinishing-hardening pressure treatment.

Research and industrial tests have shown that in most cases the fulfillment of conditions providing increased accuracy while cutting and pressure, in practice does not cause difficulties. The advantage of the combined processing method is especially clearly illustrated by the experience of combining semi-finishing and finishing processing of steel stepped rods at the "Liepajaselmash" Plant [14]. This result was convincingly confirmed by the works carried out at the Research Institute "Tractorselmash". These studies have shown that:

- combined turning and rolling treatment with differential runners with tapered rollers is successfully used instead of the final grinding operation when machining steels, cast irons, nonferrous metals and alloys with hardness up to $45 \mathrm{HRC}$;

- reducing the height of the initial bumps occurs from 10 to $0.63 \mu \mathrm{m}$;

- increase in surface hardness is up to 50\%;

- the formation and stabilization of compressive residual 
stresses favorable for most operating conditions occurs;

- the productivity of combined processing by cutting and pressure is two to seven times higher than abrasive processing, and labor intensiveness is lower than grinding.

The combination of cutting and CPD using the features and advantages of both is one of the areas for improving metalworking both in terms of shortening the processing cycle and increasing labor productivity, as well as improving the quality of machined parts.

The novelty of this research lies in the development of a mathematical model of the combined processing process. Research and development of a new model of combined machining will improve the process of finishing deep holes in thin-walled sleeves. This work and the results obtained will make it possible to recommend a combined hole machining, combining cutting and plastic deformation in practice.

\section{LITERATURE REVIEW}

Finishing, based not on cutting but on cold deformation, was recognized in 50-60 years of the last century. Issues of plastic deformation (PD) have been investigated and studied since the days of Galilei. It should be noted that the direction of these studies entered the industry primarily as the manufacture of blanks, then as a shaping, and finally as a finishing and hardening treatment. Prof. T.A. Sultanov created a whole line of science - kinetic-plastic shaping. When solving the problems of pressure processing, the engineering theory of plasticity created by E.P. Winksov and theory of metal processing created by S.I. Gubkin are used. Fundamental research has been performed on friction. Theories of friction were created by B.B. Deryagin, I.V. Kragelsky, etc., as well as many methods for determining the coefficient of friction.

Of all modern theories that explain the nature of PD and hardening of metals, the most widely recognized and most reliable theory is the dislocation theory. In modern dislocation theories, a crystal is considered, which in the initial state contains a large number of dislocations arranged in the form of a spatial grid. The existence of such grids is detected by an electron microscope, as well as by the X-ray methods. PD is represented as a process of the formation of new dislocations and their motion along the crystal. To solve the problems of SPD, the laws of least resistance, the law of constancy of volumes, the law of the least perimeter, etc., can be applied. A great contribution to the development of the scientific foundations of processing parts with SPD was made by Y.I. Barats, M.V. Kudryavtsev, E.V. Ryzhov, L.G. Odintsov [1], D.D. Papshev, Yu.G. Proskuryakov, O.A. Rosenberg [15], A.M. Rosenberg [8; 16], T.A. Sultanov, V.M. Torbilo [6], Yu.G. Schneider et al., as well as by G.A. Gazan, W. Johnson, H.I. Kleemola, et al.

In the works of A.M. Rosenberg and O.A. Rosenberg [8; 16] the cutting process in mechanics is considered as a process of CPD. The issues of strain equivalence, the possibility of the existence of a single curve of the flow of metals during cutting and other elementary deformation processes are discussed. A single fundamental mechanical characteristic of the processed material has been introduced. A theory has been created that has found wide practical application in industry in designing the process of cutting-deformation pulling. The chronological systematization of studies has shown that at present the main direction is the development of kinetic plasticity (shaping by PD), and in the field of combining processing methods, research is mainly carried out on the processes of deformation pulling and reduction.

There are patents for a combined tool that combines a cutter head and a deforming ball in its design. A number of copyright certificates for this type of instruments were registered in the 80s. Recently, new theoretical and experimental investigations have been performed. Their goal was to clarify the mechanism of CPD, to expand the capabilities of SPD methods and to improve control of these processes in order to increase the surface quality provided by them and the required operational properties of machine parts and devices, to make them more economical and reliable. In particular, such later works are those of V.M. Smelyansky, M.M. Zhasimov, Yu.G. Schneider and others. The main theoretical basis for the development of a combined axial tool can serve the works of D.D. Papshev, A.M. Rosenberg [17], L.G. Odintsov [1], D.K. Margulis, Yu.G. Proskuryakov, Yu.G. Schneider.

Noteworthy is the work of M.M. Zhasimov [11], which provides a fairly complete overview of the works in the field of studying the mechanism and laws of the distribution of contact pressures, strains, and stresses at SPD. It is concluded that it is these factors that determine changes in the surface quality of parts processed by SPD methods and almost all of its characteristics. In accordance with this, the author sets the task of establishing the laws of distribution of contact pressures, strains and stresses, taking into account the well-known position on the impossibility of establishing these laws for methods of processing with SPD. Using the theory of elasticity and plastic flow, the author proposes a methodology for determining the distribution laws, based on which it is difficult to compose the corresponding algorithm.

There is a large number of works on the distribution of axisymmetric blanks in which the issues of power loads were studied. However, in them complete solutions have only matters of distributing endless pipes with uniformly distributed internal pressure, and with the use of a number of assumptions (plane loaded state, absence of axial load, ideally plastic material). Despite all the positive features of microgeometry of surfaces processed by traditional SPD methods, it is not perfect and does not meet modern requirements for creating a qualitative theory of surfaces that provides the necessary performance properties.

\section{MATERIALS AND METHODS}

To determine the depth of work hardening of the inner surface of the deep hole of the liner, an experimental method was used. An experiment is an artificial reproduction of a phenomenon, a process under given conditions, during which a hypothesis is being tested. The control of the obtained quality parameters of the surface layer was carried out with a multifunctional device "Constant K5", a PMT-3 microhardness tester. The change in 
the structure and the depth of work hardening were recorded with an MIM-10 metallographic research microscope using the oblique section method. Also, to process the data obtained during the experiment, a mathematical method was used. Methods of data analysis and synthesis were used. These methods were used to organize the data into tables. The analysis of theoretical literature was also carried out according to the research topic.

Despite the fact that the processes of cutting and CPD are different in appearance, in reality they are based on the same physical phenomena and laws and have a number of general laws. In both processes, the total spent work is composed of the work of CPD and friction, and the share of the latter is of the same order [18]. Contact stresses on the working surfaces of the tool are also of the same order [16]. The processes of texture formation in the surface layer are identical during both cutting and PD. The friction coefficients between the working surface of the tool and the metal being machined have the same dependences on contact pressures $[16 ; 17 ; 19]$. The combination of the cutting process and SPD, as mentioned above, gives such an advantage to combined processing that it is one of the promising areas of metal processing.

Currently, deforming broaches and prefabricated constructions of rolling heads with a cutting block have found industrial use (Fig. 1). Classical combined processing is the simultaneous boring and rolling of holes with a device having a cutting (1) and rolling (2) heads with an impeller (3) located between them to prevent chips from falling under the rolling rollers. In this case, an abundant supply of cutting fluid and a mandatory exit of chips towards the velocity vector are necessary.

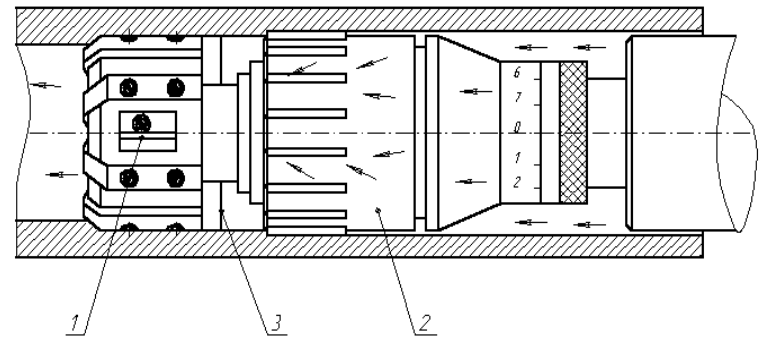

Fig. 1 Scheme of simultaneous boring and rolling

The method of smoothing for our type of parts was more appropriate from the point of view of ensuring operational characteristics, so the combination of cutting will be more effective using the principle of smoothing. Adapting to modern conditions of industrial production with a large range of processed products and frequent changes of positions, the optimal kinematic scheme of combined processing will be the classic one, including rotation and axial movement of the tool. Traditionally, a fine cutting tool for blade cutting holes is a reamer. A combined tool based on a sweep should have a deforming element that works on the principle of smoothing.

Improving the quality of machined holes with a combined tool is a task for solving which it is necessary to consider a set of issues related to a theoretical study of the PD process, determining the structural and geometric parameters of a combined cutting-deformation finishing tool, and optimizing the conditions for finishing combined processing. The hardening depth can be adjusted by changing the dimensions of the deforming tool. At a constant pressure in the contact, a decrease in the hardening depth is caused by a decrease in the contact area, and, consequently, the normal force $\mathrm{P}_{\text {norm }}$ depending on it.

\section{RESULTS}

When finishing cutting, under the action of the applied forces, PD occurs in the metal of the surface layer:

1. In the region of slip planes, the crystal lattice is distorted, in particular, its rotation near the slip planes and elastic curvature of the slip planes.

2. Slip in crystals reduces structural heterogeneities, reducing their strength. The elimination of inhomogeneities partially restores the usually reduced practical strength of the crystals, increasing the value of the critical shear stress.

3. Sliding bands divide the crystal into small parts in the form of plates, turning it into a polycrystal consisting of small elongated grains. Grinding polycrystal grains enhances the effect of blocking the slip planes of one grain by neighboring grains with a different orientation of the crystal lattice. Therefore, the slip, which began in one grain, cannot freely develop further and is hindered by neighboring grains.

4. During the slip process, the polycrystal grains are pulled in the direction of deformation, as a result of which a certain orientation of thin elongated grains is created (deformation texture).

5. When one part of the crystal slides relative to the other, on the slip planes grain fragments arise, creating a roughness of the sliding surfaces, which inhibits further movement.

6. A change in the shape of crystalline grains during deformation contributes to their mechanical engagement and impedes the mutual displacement observed during PD of polycrystals.

The totality of the listed phenomena accompanying the process of PD of polycrystals causes general hardening of the wrought metal. As a result of hardening during PD, all the characteristics of deformation resistance (yield strength, tensile strength, hardness, etc.) increase, ductility decreases (relative elongation at break and relative narrowing decrease, etc.), as well as some physical properties of the metal change. In the case of PD of a metal hardened by a previous deformation, an increase in the deformation resistance indices is noticeably slower than with a deformation of a previously not hardened metal. The degree and depth of hardening depend on the degree of PD of the metal of the surface layer, which in turn depends on the magnitude and duration of the action of external forces causing the deformation, and on the plastic properties of the deformable metal. Moreover, its structure has a great influence on the hardenability. The greatest hardening is provided by mild steels with a ferrite-perlite structure (HV 100-200) and hard steels with a martensite structure (HV 00).

Along with hardening in the metal of the surface layer, softening occurs (rest, return), partially returning the metal to 
its original properties. At room temperature, the rest of the riveted metal proceeds rather slowly or is even absent. However, when the iron is heated to $200-300{ }^{\circ} \mathrm{C}$, distortions of the crystal lattice are removed. It is important to note that metal rest occurs not only after the end of $\mathrm{PD}$, but also during the period of deformation itself. At the same time, two processes that are opposite in their results occur: hardening and softening. This can be explained by the fact that PD in a metal does not occur simultaneously in the whole volume, but begins with the most favorably oriented grains, hardening in proportion to the degree of their deformation. Only after hardening of favorably oriented grains does the least favorably oriented grains plastically deform, and PD covers the entire metal volume. At this moment, the process of softening begins in deformed and hardened grains. Preliminary processing by cutting contributes to the creation of a larger number of favorably oriented grains. Therefore, we can conclude: after the operation of the cutting part, the input of the smoothing tape is facilitated.

When machining in the cutting zone, significant cutting forces act simultaneously, creating riveting, and a temperature that causes softening of the metal. The final state of the metal of the surface layer is determined by the ratio of the processes of hardening and softening, depending on the predominance of actions in the cutting zone of power or thermal factors [20-22]. Any change in cutting conditions associated with an increase in cutting forces and the degree of PD leads to an increase in the degree of hardening. An increase in the duration of the effect of cutting forces on the metal of the surface layer leads to an increase in the depth of propagation of cold work. With combined processing, this is a positive feature.

With an increase in cutting speed, the duration of the action of deforming forces on the metal decreases, which should lead to a decrease in the depth of hardening. At the same time, with an increase in cutting speed, friction and heat generation in the cutting zone, accelerating the flow of rest, increase [23]. Therefore, with an increase in speed for metals that do not undergo structural changes when cutting, we can expect a decrease in hardening. Cutting speed, on the one hand, it is desirable to increase, because this helps to reduce heat generation; on the other hand, the depth of hardening is reduced, but this can be compensated by the number of working sealing tapes. Therefore, high speeds suit us (Fig. 2).

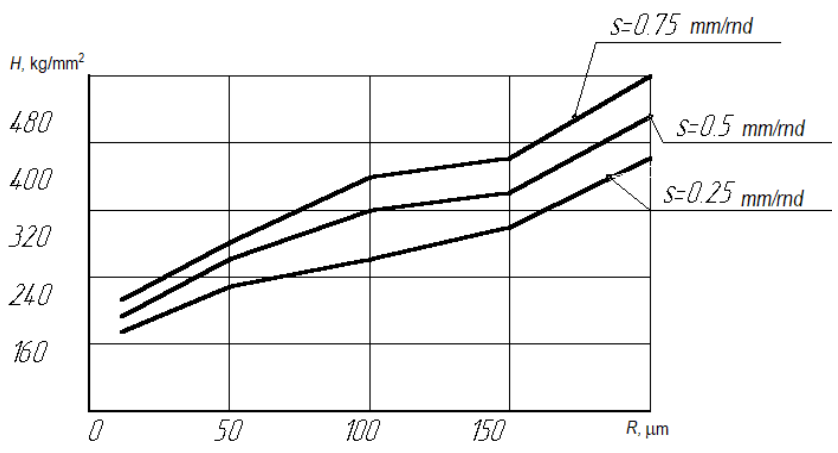

Fig. 2 The influence of the radius of blade rounding on the hardening of the metal of the surface layer
The occurrence of residual stresses in the surface layer during the machining of machine parts is explained by the following main reasons:

1. When a cutting tool acts on the surface of the processed material, PD occurs in its surface layer, accompanied by hardening and a change in some physical properties of the metal. According to M.G. Oknov [2], PD of a metal causes a decrease in its density and specific gravity, and, consequently, an increase in the specific volume, reaching, as mentioned earlier, $0.3-0.8 \%$ of the specific volume before PD. The increase in metal volume extends only to the depth of PD penetration and does not affect the metal layers lying below.

2. A cutting tool that removes elementary shavings from the surface to be machined extends the crystalline grains of the metal of the undercutting layer, which undergo elastic and plastic tensile strains in the cutting direction.

3. When separating discharge chips from the treated surface (processing of plastic metals under appropriate cutting conditions), after plastic drawing of the crystal grains of the metal of the surface layer in the cutting direction, they are additionally drawn under the influence of the chips connected to the processed surface in the direction of discharge of the chip, i.e., up. In this case, a complete reformation of the crystalline grains of the surface layer can occur (elongation in the vertical and compression in the horizontal directions) [24-26]. This leads to the appearance in the directions of the cutting speed and the supply of residual tensile stresses.

4. The heat released in the cutting zone instantly heats the thin surface layers of the metal to high temperatures, which causes an increase in its specific volume. However, no internal stresses occur in the heated layer due to the fact that with such heating of the metal, its elastic modulus decreases to a minimum, and ductility increases.

5. In the processing of metals prone to phase transformations, heating the cutting zone causes structural transformations associated, as is known, with volumetric changes in the metal. In this case, compressive stresses develop in metal layers with a structure having a larger specific volume, and residual tensile stresses arise in layers with a structure having a lower specific volume.

In combined processing, the occurrence of residual stresses after machining contributes to uniform PD.

\section{DISCUSSION}

For the study, composite samples were used having an axial line of the connector. The control of the obtained qualitative parameters of the surface layer was carried out by the "Constant K5" multifunctional device and PMT-3 microhardness tester. Changes in the structure and depth of hardening were recorded with a MIM-10 metallographic research microscope using the oblique thin section method. It was found that with an increase in tightness, the roughness parameter decreases to a certain limit (Fig. 3). With an excessively large tightness, particles of the processed metal adhere to the tool, and as a result, roughness deteriorates. Having reached a certain value, the hardening of the surface layer does not have a significant change (Fig. 4). Research and a large number of experiments made by 
A.M. Rosenberg, O.A. Rosenberg [19], V.P. Monchenko, Yu.G. Proskuryakov and others give a fairly complete picture of the influence of tightness during SPD on the roughness of the treated surface.

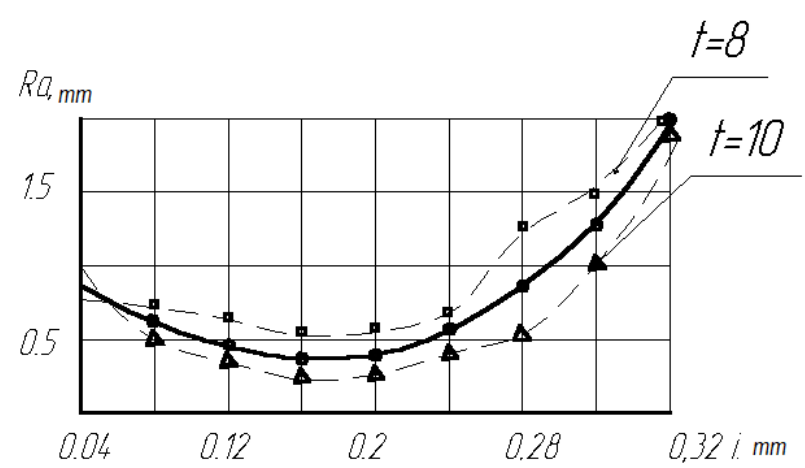

Fig. 3 The effect of absolute tightness on the roughness parameter

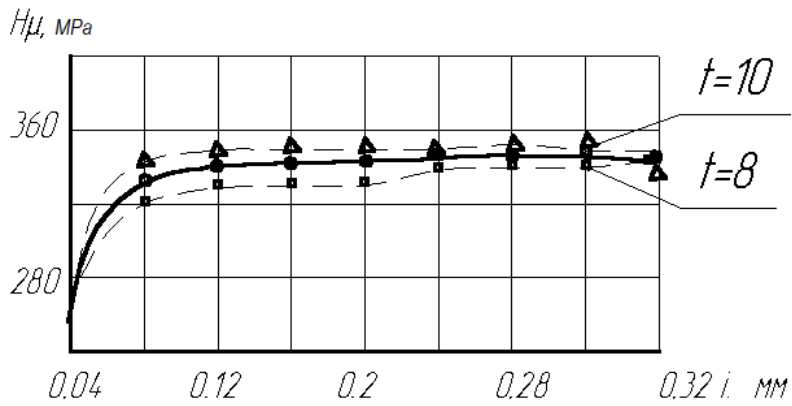

Fig. 4 The effect of tightness on the change in microhardness

As studies show, the tightness must be chosen taking into account the initial processing parameter. With an increase in tightness, the initial roughness decreases. After a critical value, improving the quality of the surface being machined is possible only in the case of initially high scallops of irregularities. With high-quality pre-treatment, quality improvement does not occur. Therefore, the pre-treatment parameter $R_{z}=12.5$ $\left(\mathrm{R}_{\mathrm{a}}=3.2 \mu \mathrm{m}\right)$ chosen by us will provide the required quality from the position of optimal operational characteristics.

The force is almost proportional to the relative tightness (more tightness - more effort is required) with single-tooth machining [27-28]. With multi-tooth machining, the force begins to decrease at a certain point. This is because the plastic wave of the metal formed as a result of additional shifts of the metal is relatively large. Consequently, a lot of work is spent on $\mathrm{PD}$, overcoming friction forces and turning surface sections (non-contact deformation zone). With a change in the mechanical characteristics of the processed material (especially the yield strength), the force changes significantly. Studies conducted by D.D. Papshev and many others, give a visual representation of the degree of surface improvement as a result of processing using SPD.

Minimum irregularities are formed at the optimum pressure, ensuring their greatest deformation and depending on the properties of the material, the dimensions of the deforming tool and the state of the initial surface. At optimal values of the normal force, the initial irregularities are completely smoothed out. A further increase in force increases plastic distortion. The height of the irregularities increases, the surface layers may collapse and cracks may appear.

The advantage of multi-tooth machining is obvious (Fig. 5). During the operation of a multi-tooth tool, the tightness is distributed between several deforming teeth; the plastic wave created by each tooth is much smaller. Since the surface layer during multi-tooth machining is hardened by each previous one, the work on the elastic deformation of the metal will be more than with single-tooth machining. However, the total work remains less. This is explained by a decrease in the parameter of surface roughness processed by previous teeth, as well as by the creation of an oriented structure of metal grains. The results of processing with a multi-tooth tool with a constant tightness value on each element are presented in Table 1.

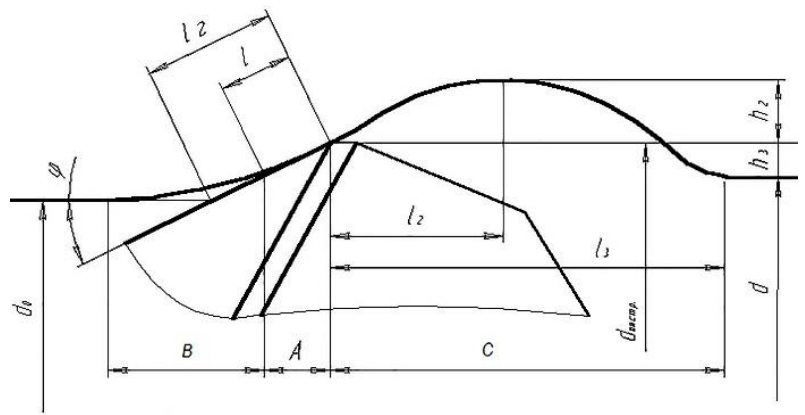

Fig. 5 Contact and non-contact deformation zone

Table 1 Dependence of the surface roughness on the tightness on the deforming element and on the total tightness

\begin{tabular}{|l|c|c|c|c|c|c|}
\hline $\begin{array}{l}\text { Tightness, } \\
\mathrm{mm}\end{array}$ & $z \mathrm{i}$ & 0.8 & $2 \times 0.4$ & $4 \times 0.2$ & $8 \times 0.1$ & $16 \times 0.05$ \\
\hline $\begin{array}{l}\text { Smoothness, } \\
\mu \mathrm{m}\end{array}$ & $R_{a}$ & 3.2 & 0.8 & 0.37 & 0.08 & 0.06 \\
\hline
\end{tabular}

It affects the effect of increased contact pressure, in connection with a decrease in tightness, an increase in the number of passes and hardening of the surface layer of the material. The optimal tightness value, which affects the height of the bumps, is selected depending on the purpose. In the case of hardening treatment, taking into account the height of the roughness of the pre-treatment $i_{\min }=2 R_{z}=20 \mu \mathrm{m}$, the maximum value of the tightness is limited by the pressure at which the plastic flow of the metal stops and fracture begins.

In a recent research paper devoted to modeling the effect of internal waves on hypothetical supports of hydraulic structures in the offshore conditions of Sakhalin Island, the authors estimate the dynamic loads on underwater vertical cylindrical parts of marine engineering structures. The authors note that the results of field observations in the South China Sea show that intense internal waves cause significantly higher loads and torques in hydraulic structures than surface waves. The authors also note the danger of such waves, therefore, the development and adaptation of models that allow assessing the risk of the impact of intense internal waves on hydraulic structures is an urgent and practically significant task [29].

Another work on a similar topic is devoted to the study of 
convective heat transfer in flat plate solar collectors. The authors note that, as can be seen from the analysis of the study of heat transfer by round and flat tubes with the coordination of forced and free convection, they are placed vertically or horizontally with different fluids flow directions. The researchers obtained the dependences of the Nusselt criterion in round and flat pipes, which show that the corresponding equations allow one to determine the heat exchange rate for all liquids with appropriate accuracy. The authors also studied the Prandtl criterion for liquids, which plays an important role in the placement of points in relation to the curve [30].

\section{CONCLUSION}

It has been established that one of the most promising areas of metalworking is the use of combined tools on generalpurpose machines. They make it possible to perform blade pretreatment and reinforcing surface deformation on a workpiece in one working stroke. The complexity of the process and the lack of a mathematical model (which would make it possible to satisfactorily calculate its characteristics: deformations, forces, contact pressures, etc.) allow us to talk about the insufficient degree of research and the relevance of this scientific and engineering problem. The following solutions to the main research tasks were proposed:

- development of a new effective process for machining cylinder bores of hydraulic equipment, combining cutting and PD of the surface layer with an axial tool;

- a theoretical study of the technological laws of the process, which can be the basis for the design of tools and technological processes of combined processing;

- an experimental study of the influence of design parameters of a combined tool and processing modes on surface quality;

- an experimental study of the influence of the main processing parameter - tightness on the change in the dimensions of the part and determination of technological dependencies;

- development of methods for calculating the parameters of combined hole processing;

- development of new designs of combined tools with sealing ribbons based on the complete research.

The experimental studies of the material and its properties acquired as a result of combined processing showed that:

- the effect of hardening of the surface layer of the treated hole is observed at a depth of 1-1.2 mm;

- the surface layer of the machined hole acquired a hardness of 30 HRC;

- the structure of the deformed layer (especially at the surface) acquires a pronounced fibrous character.

A formalized model of the combined process of machining cylinder bores for hydraulic equipment has been developed, taking into account the features and trends of modern metalworking. This model quantitatively connects the magnitude of the normal force with the physical-mechanical properties of the workpiece, technological conditions and design parameters of the tools.
It has been experimentally established that the optimal roughness of the preliminary processing of the holes, which ensures the subsequent high-quality processing of the hole, can be obtained with a tool having a principal angle of $6 \square$. It is established that the shape of the sealing tape of the combined tool should be as close as possible to the radius. The radius of the deforming element determines the degree of hardening and the quality of the surface layer. Approximating dependences of the calculation of the main parameter of the combined processing (the tightness vs the wall thickness of the part) are found.

Based on a theoretical study of contact pressure, it was found that in the process of combined processing, all factors that increase contact pressure contribute to a decrease in the surface layer roughness. It was experimentally established that the feed during combined processing is a determining factor in the quality of processing. The height of the bumps is directly proportional to the amount of feed squared. It was found that during combined hole processing, the processing speed does not significantly affect the quality of the surface layer, but is a limiting factor in heat generation. It should be borne in mind that the distribution of the allowance for deformation for each tooth in the axial section may be the subject of independent research.

The development of theoretical principles and the creation on their basis of a new process for processing holes with predictable properties has become possible because of the integrated use of theoretical and experimental research methods. The solution of a number of new problems posed in the work became possible due to the well-known achievements of the theories of cutting, elasticity, PD and does not contradict their provisions. It is based on rigorously proven conclusions from fundamental and applied sciences, such as mathematical analysis, mathematical statistics, theoretical mechanics, optimization theory, and experimental planning.

This research provides an opportunity to improve the finishing process for deep holes in thin-walled liners. The results of this study allow us to recommend a combined hole machining, combining cutting and plastic deformation. In the course of the research, a mathematical model of the combined processing process was obtained. This work will improve the finishing process, and the developed mathematical model has practical and theoretical scientific value.

\section{References}

[1] L.G. Odintsov, Finishing parts with Diamond Smoothing and Vibration Smoothing. Moscow: Mashinostroyeniye, 1985.

[2] N.N. Zorev, Questions of Mechanics of Metal Cutting Process. Moscow: Mashinostroyeniye, 1976.

[3] E.A. Chudakov (Ed.), Engineering. Encyclopedic Reference Book. Volume 1. Book 1. Moscow: Mashinostroyeniye, 1995.

[4] A.I. Levin, Mathematical Modeling in Research and Design of Machine Tools. Moscow: Mashinostroyeniye, 1978. 
[5] E. Thomsen, Sh. Young, Sh.O. Kobayashi, Mechanics of Plastic Deformation in Metal Processing. Moscow: Mashinostroyeniye, 1979.

[6] V.M. Torbilo, Diamond Smoothing. Moscow: Mashinostroyeniye, 1972.

[7] G.M. Azarevich, E.V. Kirsanova-Belova, B.I. Akimov, "Combination of cutting processes of surface plastic deformation during automated turning," Eng. Bull., vol. 1, 1985, pp. 46-49.

[8] A.M. Rosenberg, O.A. Rosenberg, E.K. Posvyatenko, M.I. Polishchuk, "Deformation cutting broach," Mach. Builder, vol. 12, 1973, pp. 31-32.

[9] G.G. Dobrovolsky, I.A. Motovilovets, "Calculation of the temperature in the zone of contact of the tool with the product during deformation drawing in order to select tool parameters and processing modes," Phys. Phenom. Deforming Drawing Cutting Ductile Met., 1972, pp. 5256.

[10]N. John, F. Lyon, Statistics and Experimental Design in Engineering and Science. Data Processing Methods. Moscow: Mir, 1980.

[11]M.M. Zhasimov, Quality Management of Parts with Surface Plastic Deformation. Alma-Ata: Nauka, 1986.

[12] I.P. Zakharenko, Fundamentals of Diamond Processing of Carbide Tools. Kiev: Naukova dumka, 1981.

[13] G.G. Inozemtsev, Design of Metal Cutting Tools. Moscow: Mashinostroyeniye, 1984.

[14]E.M. Chistyakov, A.A. Shepelev, T.M. Duda, V.P. Chernykh, Tool Made of Metallized Superhard Materials. Kiev: Naukova dumka, 1982.

[15] O.A. Rosenberg, The Mechanics of the Interaction of the Tool with the Product during Deformation Drawing. Kiev: Naukova dumka, 1981.

[16] A.M. Rosenberg, O.A. Rosenberg, "On the distribution of pipe billets by internal pressure," Appl. Mech., vol. 10, 1976, pp. 133-137.

[17] A.M. Rosenberg, A.N. Eremin, Elements of the Theory of Metal Cutting. Moscow: Mashgiz, 1976.

[18] R.M. Mukhamadeyeva, Improving the quality of Hole Processing with Combined Cutting-Deforming Reamers. Omsk: Publishing House of OmSTU, 2005.

[19] A.M. Rosenberg, O.A. Rosenberg, Yu.F. Busel, A.D. Kritsky, "The role of lubricant in the process of deforming drawing," Synth. Superhard Mater. Hard Alloys, vol. 3, no. 4, 1974, pp. 240-249.

[20] I.I. Kichkin, E.P. Skornyakov, Patent Studies in the Course and Diploma Design in Higher Educational Institutions. Moscow: Vysshaya shkola, 1979.

[21]A.D. Kritsky, Features of the Development of Carbide Deforming Broaches for Machining Holes in Parts of Difficult to Process Metals and Alloys. Kharkov: Kharkov Aviation Institute, 1983.

[22] G.R. Kruglov, "The shock method of forming regular microreliefs on the surfaces of machine parts and devices," Collect. LDNTP Technol. Support Control Regul. Microrelief Mech. Eng., 1984, pp. 22-30.

[23] V.A. Kroha, Hardening of Metals during Cold Plastic Deformation. Moscow: Mashinostroyeniye, 1980.
[24] V.S. Kushner, Thermomechanical Theory of the Process of Continuous Cutting of Plastic Materials. Irkutsk: Publishing House of Irkutsk University, 1982.

[25] A.I. Levin, Mathematical Modeling in Research and Design of Machine Tools. Moscow: Mashinostroyeniye, 1978.

[26] A.A. Matalin, Technological Methods to Increase the Durability of Machine Parts. Kiev: Tekhnika, 1981.

[27]R.M. Mukhamadeyeva, "Development prospects of introduction of innovative technologies 3dprinting in Kazakhstan,” OP Conf. Ser.: Mater. Sci. Eng., vol. 709, 2020, $044080 . \quad$ Available: https://iopscience.iop.org/article/10.1088/1757899X/709/4/044080/meta

[28] M.A. Berikbaeva, B.T. Khairullin, R.M. Mukhamadeyeva, "Combined hole processing for hydraulic cylinders," in LXVI Int. Sci.-Pract. Conf. "Int. Sci. Rev. Probl. Prospects Mod. Sci. Educ.”, Boston, 2020, pp. 7-11.

[29] E. Rouvinskaya, O. Kurkina, A. Kurkin, A. Zaytsev, "Modeling of internal wave impact on hypothetical pillars of hydraulic engineering constructions in the conditions of the Sakhalin island shelf," WSEAS Trans. Fluid Mech., vol. 13, 2018, pp. 101-107.

[30] Ye. Amirgaliyev, M. Kunelbayev, A. Kalizhanova, B. Amirgaliyev, A. Kozbakova, O. Auelbekov, N. Kataev, "Study of convective heat transfer in flat plate solar collectors," WSEAS Trans. Syst. Control, vol. 14, 2019. pp. 129-137.

\section{Contribution of individual authors to the creation of a scientific article (ghostwriting policy)}

Meruert Berikbaeva and Railya Mukhamadeyeva conceived of the presented idea. Beibyt Khairullin formulated research issues and hypothesis of presented problem and designed the research. Railya Mukhamadeyeva methodically managed and organized experiments and evaluated results. Meruert Berikbaeva and Beibyt Khairullin conducted experiments. All authors discussed the results and contributed to the final manuscript.

\section{Creative Commons Attribution License 4.0 (Attribution 4.0 International , CC BY 4.0)}

This article is published under the terms of the Creative Commons Attribution License 4.0

https://creativecommons.org/licenses/by/4.0/deed.en_US 